\title{
The safety, efficacy and cost-effectiveness of the Maxm Skate, a lower limb rehabilitation device for use following total knee arthroplasty: study protocol for a randomised controlled trial
}

\author{
Matthew G. Liptak ${ }^{1 *}$, Annika Theodoulou ${ }^{2,3,4}$, Billingsley Kaambwa ${ }^{5}$, Steve Saunders ${ }^{6}$, Scott W. Hinrichs ${ }^{7}$,
} Richard J. Woodman ${ }^{8}$ and Jeganath Krishnan ${ }^{2,3}$

\begin{abstract}
Background: Physical rehabilitation is required to enhance functional outcomes and overall recovery following total knee arthroplasty (TKA). However, there are no universally accepted clinical guidelines available to consistently structure rehabilitation for TKA patients. A common method is rehabilitation provided in an outpatient setting, on a one-to-one treatment basis. This method is resource-intensive and outcomes must be compared to less costly alternatives such as home-based rehabilitation. The current study will analyse a novel home-based rehabilitation program. The Maxm skate is a portable, lower-limb, postoperative, rehabilitation exercise device for individual use in a hospital or home-based setting. This study was developed to compare the safety, efficacy and cost-effectiveness of the Maxm Skate rehabilitation program to standard rehabilitative care following TKA. The primary outcome is the range of motion (ROM) achieved by patients who received the Maxm Skate program compared to standard care at three months post TKA. Secondary outcomes include patient-reported outcomes, costs and functional evaluations which will be collected at multiple time-points up to 12 months after TKA.
\end{abstract}

Methods: This is a single-blinded, randomised controlled trial (RCT) in which 116 eligible participants consented for primary TKA will be randomly allocated to receive either the Maxm Skate rehabilitation program or standard rehabilitative care. Fifty-eight participants per group will provide $90 \%$ power $(a=0.05)$ to detect $10^{\circ}$ of difference in ROM between groups at three months after TKA, assuming a within-group standard deviation of $16^{\circ}$ and allowing for $5 \%$ loss to follow-up. Participants randomised to the Maxm Skate group will use the skate device and accompanying iOS App and sensors to complete rehabilitation exercises, as outlined in the Maxm Skate Rehabilitation Guide. Outcomes will be compared to those receiving standard rehabilitative care. A blinded physiotherapist will evaluate functional outcomes preoperatively and at 2, 4, 6, 12, 26 and 52 weeks after TKA. The functional assessment will include measures of knee ROM, pain, isometric knee strength, balance and knee/thigh circumference. Limited measures will also be assessed at day 2 postoperatively by an alternate, unblinded physiotherapist. Clinical outcome measures will be administered preoperatively and at 6, 12 and 52 weeks postoperatively. An economic evaluation will be conducted and participants will be screened for adverse event occurrences from the time of consent to 12 months postoperatively.

(Continued on next page)

\footnotetext{
* Correspondence: liptakadmin@orthosa.com.au

'Orthopaedics SA, Adelaide, South Australia, Australia

Full list of author information is available at the end of the article
}

(c) The Author(s). 2019 Open Access This article is distributed under the terms of the Creative Commons Attribution 4.0 International License (http://creativecommons.org/licenses/by/4.0/), which permits unrestricted use, distribution, and reproduction in any medium, provided you give appropriate credit to the original author(s) and the source, provide a link to the Creative Commons license, and indicate if changes were made. The Creative Commons Public Domain Dedication waiver (http://creativecommons.org/publicdomain/zero/1.0/) applies to the data made available in this article, unless otherwise stated. 
(Continued from previous page)

Discussion: This RCT will be the first to investigate the safety, efficacy and cost-effectiveness of the home-based Maxm Skate Rehabilitation program, in comparison to standard rehabilitative care following primary TKA.

Trial registration: Australian New Zealand Clinical Trials Registry, ACTRN12616001081404p. Registered on 11 August 2016.

Keywords: Total knee arthroplasty, Rehabilitation, Home-based, Range of motion, Cost-effectiveness

\section{Background}

Primary total knee arthroplasty (TKA) is an increasingly common surgical intervention used to alleviate pain and physical dysfunction associated with end-stage degenerative joint disease. Following surgery, patients experience lower-extremity muscle weakness and commonly require physical rehabilitation to enhance functional outcomes and overall recovery $[1,2]$. While TKA is a successful procedure for many patients, up to $15-20 \%$ of patients remain unsatisfied $[3,4]$. Limited postoperative range of motion (ROM), which is often associated with arthrofibrosis, may be one factor associated with patient dissatisfaction. The recorded incidence of arthrofibrosis after primary TKA varies in the literature, in the range of approximately $1-13 \%$ [4-6]. When it occurs, it is a significant cause of patient dissatisfaction associated with poor ROM and is a causative factor of revision TKA $[5,6]$. Therefore, as limited ROM is an early indicator of potential arthrofibrosis, rehabilitation that enables ongoing flexion/extension exercises and ongoing collection of such progress, may improve early detection of arthrofibrosis and as such appropriate rehabilitative management before the need for revision surgery. In addition, it is well established that daily activities including climbing stairs and standing from a chair require $90-120^{\circ}$ of flexion, kneeling and squatting $110-165^{\circ}, 135^{\circ}$ of flexion to lift from a bath and $>150^{\circ}$ for yoga or gardening [7-9]. However, patients rarely achieve $>120^{\circ}$ of flexion post TKA and $110^{\circ}$ of flexion is an optimal goal for rehabilitation following TKA [9-11]. At present, there are no universally accepted or widely implemented clinical guidelines available to consistently structure patient rehabilitation following TKA [12-14]. Consequently, rehabilitative protocols are not standardised and regularly based on institution-, surgeon- or occasionally, patientspecific preferences $[1,2,12]$. This lack of uniformity in the mode of rehabilitation results in therapy of different types, frequency, intensity and duration across sites and countries $[1,2]$.

In Australia, rehabilitation most commonly provided is in an outpatient setting, on a one-to-one treatment basis [12]. Outpatient physiotherapy in a clinic-based setting is beneficial as a physiotherapist can monitor progress and modify therapy; however, such methods are resource-intensive and impose a significant cost burden [2]. Further concerns include patient transportation to clinics following surgery and accessibility to qualified rehabilitative specialists.

In 2016, the number of primary TKA procedures performed in Australia increased by 139.8\% since 2003 and by $2.8 \%$ compared to 2015 [15]. Such trends suggest that TKA use will continue to rise, bringing concern of the sustainability and economic impact of one-to-one rehabilitation commonly employed. There is a need to determine whether such outpatient physiotherapy yields superior outcomes compared to less costly alternate forms such as group-based, home-based or tele-rehabilitation.

Several randomised controlled trials (RCT) have considered the influence of outpatient physiotherapy on functional outcomes following TKA. Mockford et al. [16] and Rajan et al. [17] both concluded no significant difference in ROM achieved between patients who did and did not receive outpatient physiotherapy 12 months after TKA. However, methodologically short-comings, and limited descriptions of the standard outpatient physiotherapy provided, caused a literature reviewer to suggest that conclusions from these studies were not supported [2]. A methodologically robust RCT found that one-to-one outpatient treatment provided over a six-week period did not provide superior self-reported or performance-based outcomes, compared to groupbased or monitored home programs up to 12 months after TKA [18]. Furthermore, a recent meta-analysis demonstrated no difference in knee ROM and shortterm functional improvements between outpatient physiotherapy in a clinic-based setting and non-supervised home-based exercise regimens following discharge after TKA [19].

The common provision of one-to-one therapy is not currently supported over more economical home-based rehabilitative methods of which elicit similar self-reported, functional and performance-based outcomes $[18,20]$. The current study will analyse a novel homebased rehabilitation program as compared to the standard rehabilitative care options available to patients. As detection of arthrofibrosis is typically first observed through reduced postoperative $\mathrm{ROM}$, it is particularly important to this study to collect ROM data. Furthermore, early detection of poor ROM (three months) may enable implementation of appropriate rehabilitation 
before the requirement of manipulation under anaesthesia (MUA) or revision surgery.

The Maxm Skate device is a portable, lower-limb, postoperative and post-injury rehabilitation exercise device for individual use in a hospital or home-based setting. The Maxm Skate intends to facilitate rehabilitation and conditioning of the lower limb through graded therapeutic exercise with the aim to promote tissue healing, remodelling and strengthening. It allows the patient to perform strengthening exercises with minimal joint loading during their rehabilitation period.

The Maxm Skate package comprises the Skate device and rope (for assisted active motion and resistance training), sensors, iOS Application (App) and website. The Skate device is accompanied by two sensors and a mobile App which are designed to provide real-time, objective data on exercise and rehabilitation progress, in particular ROM following each home-based exercise therapy session. This sensor technology also enables the clinician to monitor accurate compliance and ROM data (Flexion and Extension) remotely.

This clinical trial is the first to compare the safety, efficacy and cost-effectiveness of the Maxm Skate rehabilitation package to standard rehabilitative care up to 12 months after TKA.

\section{Objectives}

The primary objective of this RCT is to assess the ROM achieved by TKA patients whom received the Maxm Skate rehabilitation package compared with standard rehabilitative care, three months postoperatively.

Secondary objectives include the comparison of functional, clinical and performance-based outcome measures between the Maxm Skate and standard rehabilitative care groups at multiple times points, up to 12 months after TKA. An economic evaluation assessing the relative costeffectiveness of the Maxm Skate rehabilitation package compared to standard care will be conducted; patients will be screened for adverse event (AE) occurrences and complications from the time of consent to one year postoperatively.

\section{Methods/Design}

This is a single-blinded RCT comparing the safety, efficacy and cost-effectiveness of the Maxm Skate Rehabilitation Program to standard rehabilitative care following TKA.

\section{Study setting}

The study will be coordinated by the International Musculoskeletal Research Institute Inc. at the study site, Flinders Private Hospital (FPH), Bedford Park, South Australia.

\section{Recruitment and selection criteria}

Consecutive patients consented for elective primary TKA at a single orthopaedic surgeon's private clinic will be screened for eligibility. The treating surgeon (MGL) will screen all patients against the selection criteria and document reasons for ineligibility in the patient screening log. Study inclusion criteria include:

1. The patient requires a primary TKA due to non-inflammatory degenerative joint disease (e.g. osteoarthritis and traumatic arthritis) or inflammatory joint disease (e.g. rheumatoid arthritis).

2. The patient must understand the conditions of the study and be willing and able to provide written informed consent.

3. The patient is a skeletally mature man or a nonpregnant woman, aged $\geq 30$ years.

4. The patient agrees to comply with the specified preoperative and postoperative study requirements.

Study exclusion criteria include:

1. The patient has an emotional or neurological condition that would pre-empt their ability to participate in the study including mental illness, intellectual disability and drug or alcohol abuse.

2. Any patient who is unable to meet the requirements of the use of the Maxm Skate Rehabilitation Device.

3. The patient is unable to perform home exercise program without supervision or assistance.

A clinical nurse or authorised delegate will invite eligible patients to come in to discuss the study. Patients will be informed of the study purpose and the potential risks and benefits known, or that can be reasonably predicted, as outlined in the Participant Information Sheet.

The patient will then be given the opportunity to ask any clarifying questions and invited to complete the consent form, indicating their understanding of the study and consent for participation. Following consent, participants will be randomised and baseline measures obtained. The randomisation number will be used to provide anonymous identification of the participant on study documents from there forth.

\section{Randomisation and blinding}

The randomisation schedule will be prepared using numbered and sealed, opaque envelopes. Participants will be randomly allocated in a 1:1 ratio to either the 'Maxm Skate Rehabilitation Program' or the 'Standard Rehabilitative Care' group. Block randomisation in groups of four will be used to ensure balance in the two groups. Randomisation will also be stratified by gender to ensure balance between groups for each gender. We 
note that the number of women undergoing TKA for osteoarthritis has been shown to be greater in Australia [21]. In addition, studies have demonstrated that there are differences between men and women in muscle and physical function recovery after TKA [22, 23].

The study will be single-blinded with blinding of study investigators but not participants. Participant randomisation to either Maxm Skate or Standard Care will be performed by the unblinded Study Coordinator, who will hold the treatment allocation code in confidence. The statistician will be blinded to the treatment allocation code when performing all analyses. Functional evaluations and performance tests will be conducted by a trained physiotherapist $(\mathrm{SWH})$ who will be blinded to group allocation. This blinded physiotherapist will conduct all functional evaluations, excluding postoperative day 2, due to practical constraints. Non-blinded wardbased physiotherapists will conduct the day 2 assessments, as well as the preoperative Maxm Skate education session for participants allocated to the Maxm Skate group.

Due to the nature of the intervention there is a potential for participants to disclose, or physiotherapist to gauge, which group the participants are assigned. Participants will be educated and instructed not to advise the physiotherapist or treating surgeon of their group assignment at the time of their consent and randomisation with the unblinded Study Coordinator. However, if participants are concerned about their rehabilitative progress and feel it is necessary to discuss this with their treating surgeon, they will be able to do so. The breaking of blinding will be collected and reviewed during data analysis.

\section{Interventions}

All participants will undergo primary TKA at Flinders Private Hospital. All hospital TKA patients are invited to attend a 'Joint replacement information session' which provides further education and the opportunity for physiotherapy and nursing staff to address patient queries. An exercise booklet is issued describing the postoperative exercises and patients are asked to familiarize themselves with the exercises.

\section{Maxm Skate rehabilitation program}

Participants randomised to the Maxm Skate rehabilitation program will be required to use the skate device and accompanying iOS App and sensors to complete rehabilitation exercises, as outlined in the Maxm Skate Rehabilitation Guide (see Additional file 1). Participants will be asked to download the Maxm Skate App on an iOS device, which will be provided if a personal device is not available.
Clinical data will be collected via the App aligns with the exercises outlined in the rehabilitation guide, including exercises completed, number of repetitions, length of time completing exercise and outcomes achieved. Data collected via the App are stored in the Google Firestore cloud database and within the iOS device itself.

Preoperative phase Participants will attend a preoperative device education session where they will be introduced to the Maxm Skate program by a physiotherapist. Participants will be given a description of the functionality, set up and safe usage of the Maxm Skate device and sensor, and educated on the Maxm Skate exercise program. Following this presentation, participants will be issued with a rehabilitation guide to familiarise themselves with the postoperative exercise program and given the opportunity to trial the skate package.

Postoperative inpatient phase In the inpatient acute setting, the Maxm Skate group will receive the Maxm Skate in addition to standard care as outlined above for the Standard Care group. The Maxm Skate group may access inpatient rehabilitation if clinically indicated.

Postoperative outpatient phase Following the inpatient program, participants in the Maxm Skate program will only receive rehabilitation provided through the Maxm Skate program, described in the Maxm Skate Rehabilitation Guide (see Additional file 1). This program includes functional assessment checks at two weeks and six weeks at the participant's home. This will ensure participants are fit to progress through from Stage One to Stage Two and from Stage Two to Stage Three of the program. Additional functional checks will be conducted if required, as assessed by hospital staff at discharge from hospital. Furthermore, the checks will allow early identification of participants who are not advancing and may require additional rehabilitative support.

Participants that are identified as requiring additional physiotherapy will receive such treatment, as required. Participant functional progress will also be assessed by the orthopaedic surgeon at the six-week orthopaedic follow-up appointment. Any additional outpatient physiotherapy received by a participant randomised to the Maxm Skate program will be permitted and recorded in the participant study file.

\section{Standard rehabilitative care}

Inpatient physiotherapy care provided to patients in hospital will include undertaking the following standard care exercises: ankle pumps; static quadriceps; supine knee flexion; inner range quadriceps; straight leg raise; passive knee extension; seated assisted knee flexion; and active 
knee extension in sitting. Once discharged from hospital, physiotherapy follow-up varies on an individual basis. The patient is provided four patient-specific options which are described in the Outpatient Standard Care Physiotherapy Protocol (FPH) (see Additional file 2).

\section{Study outcomes and participant timeline}

The primary objective is ROM achieved at three months after TKA. Secondary outcomes include patient-reported outcomes, costs and functional evaluations which will be collected at multiple time-points up to 12 months after TKA. Patients will be screened for AE occurrences and complications from the time of consent to one year postoperatively. The study assessment schedule in outlined in Fig. 1.

\section{Baseline and operative data}

Demographic details, knee pathology and medical history will be collected preoperatively by the clinical nurse or research delegate.

In order to limit any potential variability attributed to the prosthetic device, a single total knee prosthesis, the Advanced Coated System ACS $^{\circ}$ Fixed Bearing System (Oceania Orthopaedics Pty. Ltd.) will be implanted in all study participants by a single orthopaedic surgeon (MGL). The medical device will be implanted using a medial para-patella surgical approach, using both a cemented and cementless technique depending on patient characteristics and surgeon discretion. A midline skin incision will be conducted for all patients and device-specific instrumentation will be used.

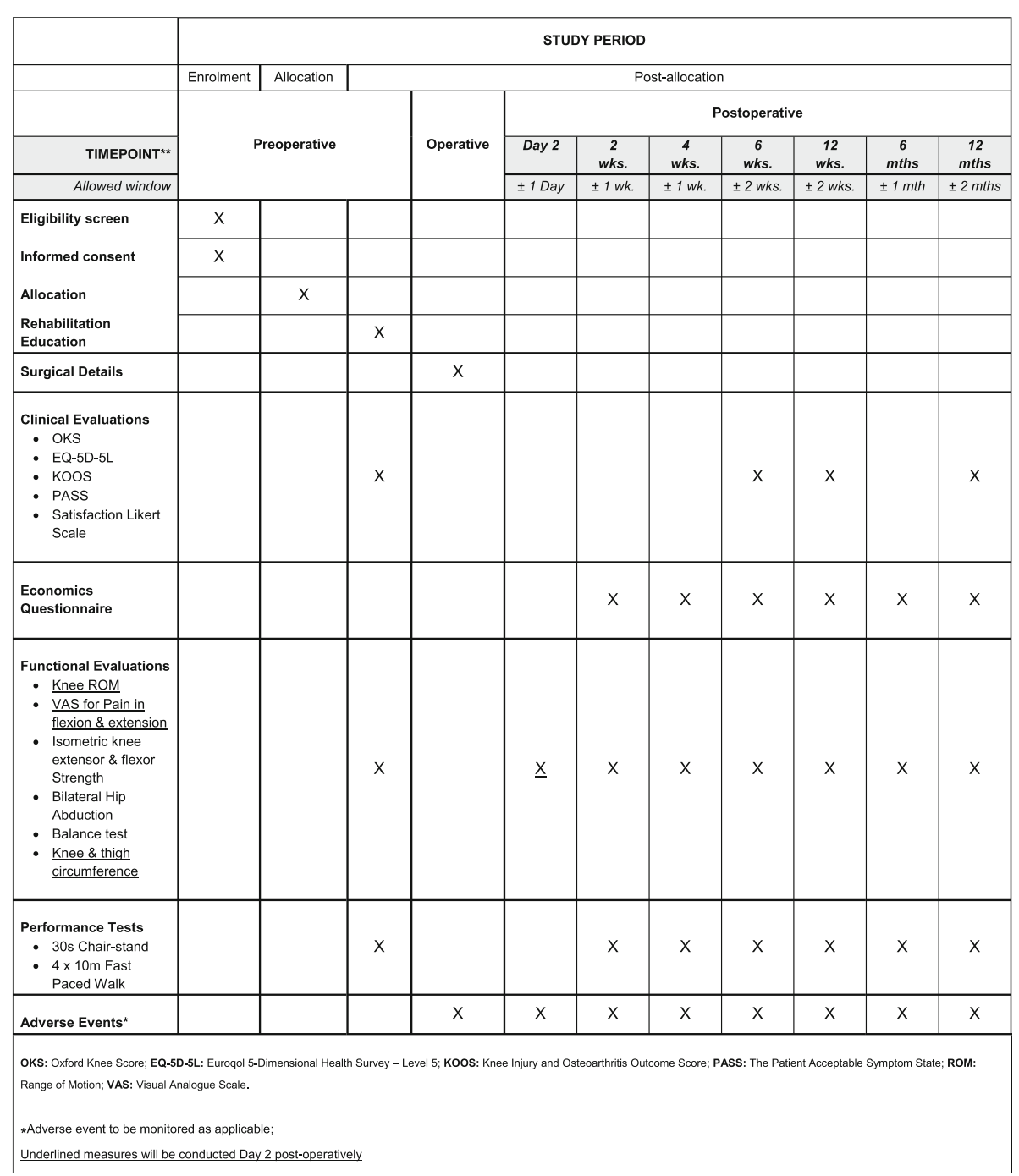

Fig. 1 Schedule of events 


\section{Patient-reported outcome measures}

Patient-reported outcome measures will be assessed for each group preoperatively and postoperatively at six weeks, 12 weeks and 12 months. The Oxford Knee Score (OKS) [24] and Knee Injury and Osteoarthritis Outcome Score (KOOS) [25] will be used to assess knee-specific symptoms, pain and function, while the Euroqol 5 Dimensional Health Survey - Level 5 (EQ-5D-5L) [26] will be used to survey health status. The Patient Acceptable Symptom State (PASS) [27] will be used to assess whether the patient feels that they are at a satisfactory state. Lastly, level of satisfaction with the outpatient rehabilitation received will be measured using a 5-point Likert scale as follows: 'very unsatisfied'; 'unsatisfied'; 'neither satisfied nor unsatisfied'; 'satisfied'; 'very satisfied'.

Participants in the Maxm Skate group will also be required to complete a short questionnaire regarding their participation in the Maxm rehabilitation program. This form will be administered at a visit with the Study Coordinator either before or after each functional evaluation with the blinded physiotherapist.

\section{Functional evaluations}

The following functional evaluations will be performed by a blinded physiotherapist preoperatively and at 2, 4, 6, 12, 26 and 52 weeks after TKA. Some measures will also be assessed at day 2 postoperatively; however, these measures will be taken by an unblinded ward physiotherapist. Functional evaulations are illustrated in Fig. 2.

Joint range of motion and the Visual Analogue Scale for Pain in flexion and extension In a seated position, active joint ROM in flexion and extension will be measured using a standard goniometer. Furthermore, pain at end-of-range flexion and extension will be measured using the Visual Analogue Scale (VAS) for Pain. Recovery in knee ROM has been found to plateau 12 months after TKA, therefore end-of-study has been selected to be 12 months postoperatively [28]. Both measures will also be assessed at day 2 after TKA.

Isometric knee extensor strength, flexor strength and bilateral hip abduction Hand-held isometric [29-32] and isokinetic [30, 31, 33, 34] dynamometry has previously
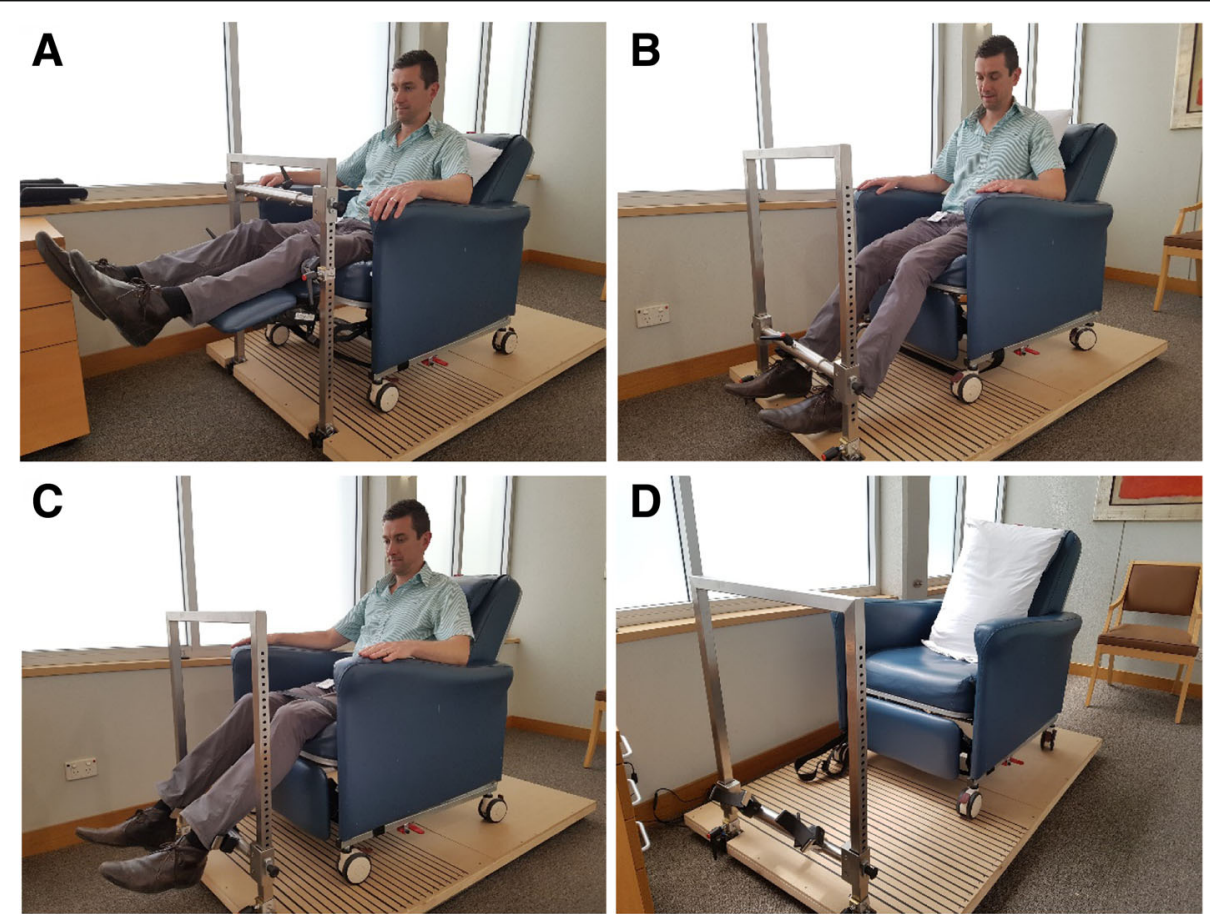

Fig. 2 KangaTech set-up. a Hip abduction: for bilateral hip abduction testing, the participant will also be seated, with the knee flexed at $15^{\circ}$ and lumbo-pelvic-hip complex flexed at $60^{\circ}$. The dynamometry pad will be positioned at the level of the lateral femoral condyles. b Knee extension: unilateral knee extension performed with participants seated, their knee at $45^{\circ}$ flexion and lumbo-pelvic-hip complex at $60^{\circ}$ flexion. The dynamometry pad will be positioned at the level of the lateral malleolus at right angles to the shank/tibia. Each thigh will be secured to the seat with a belt above the level of the surgical incision (> $10 \mathrm{~cm}$ above the supra patella border). c Knee flexion: unilateral knee extension performed with participants seated, their knee at $45^{\circ}$ flexion and lumbo-pelvic-hip complex at $60^{\circ}$ flexion. The dynamometry pad will be positioned at the level of the lateral malleolus at right angles to the shank/tibia. Each thigh will be secured to the seat with a belt above the level of the surgical incision $(>10 \mathrm{~cm}$ above the supra patella border). $\mathbf{d}$ Set-up alone at Flinders Private Hospital for use in this study 
been used to safely and effectively measure maximal quadricep and hamstring muscle strength in $\mathrm{OA}$ and TKR populations. In the present study, a customised fixed-frame portable dynamometry hardware and software system (KangaTech, Melbourne, Australia) will be used to measure maximum voluntary isometric strength of the quadriceps, hamstring and hip abductor muscle groups of each participant.

Maximum voluntary isometric contraction (MVIC) will be tested for the hip abductors, knee extensors and knee flexors in a seated position to maximise participant comfort. Unilateral knee extension and flexion tests will be performed with participants in a seated position with their knee at $45^{\circ}$ of flexion and with their lumbo-pelvic-hip complex at $60^{\circ}$ of flexion. The dynamometry pad will be positioned at the level of the lateral malleolus at right angles to the shank/tibia. Each thigh will be secured to the seat with a belt above the level of the surgical incision ( $>10 \mathrm{~cm}$ above the supra patella border). For bilateral hip abduction testing, participants will also be seated, with the knee flexed at $15^{\circ}$ and lumbo-pelvic-hip complex flexed at $60^{\circ}$. The dynamometry pad will be positioned at the level of the lateral femoral condyles.

Participants will perform each test in a randomly selected order after having had three gradually ramped practice/warm-up trials. During these trials, participants will be asked to produce an isometric contraction at $20 \%, 50 \%$ and $80 \%$ of their perceived maximum effort for $3 \mathrm{~s}$. Participants experiencing any discomfort that is unusual and/or with an associated pain score of $>3 / 10$ (measured by the VAS - Pain) above their resting level of pain will be asked not to proceed beyond this level of force or effort. In this case, the pain limited MVIC will be recorded.

Participants that warm up without the forementioned symptoms will proceed to $2 \times 5 \mathrm{~s}$ MVIC test trials for each test. Participants will again be advised to limit force in the presence of discomfort. Time allowed for recovery between all tests will be $20 \mathrm{~s}$.

Balance test, knee circumference and thigh circumference This balance test will be performed in a single-leg standing position, with eyes open. The ability to maintain balance in this position will be recorded in seconds.

The participant's knee and thigh circumference will be measured using a regular tape measure. The measurements will be taken at two points, specifically at the superior pole of the patella and $15 \mathrm{~cm}$ above the superior pole of the patella. Both measures will also be assessed at day 2 after TKA.

Clinical performance tests Clinical performance tests, including the 30-s Chair-Stand Test and the $4 \times 10 \mathrm{~m}$
Fast Paced Walk Test, will be performed in accordance with the Osteoarthritis Research Society International (OARSI) Report guidelines [35]. The tests will be conducted preoperatively and at 2, 4, 6 and 12 weeks, 6 and 12 months postoperatively.

\section{Economic evaluation}

An economic evaluation assessing the relative cost-effectiveness of the Maxm Skate rehabilitation device compared to standard care will be conducted.

This analysis will take the form of an economic evaluation comparing the relative cost-effectiveness analysis of the Maxm Skate rehabilitation device to standard care using data collected during the trial. The primary outcome will be the incremental cost per unit increase in knee ROM while a secondary outcome will be the incremental cost per quality-adjusted life year (QALY) gained over the period of the primary study and based on the EQ-5D-5 L. As an Australian scoring algorithm for the EQ-5D-5 L is not yet available, the UK value set and scoring algorithm [36] will be used to convert the individual responses to the EQ-5D-5 L at each time point. Resource use associated with each trial arm will be combined with unit costs for these resources in order to estimate total costs in the trial. Costs will be estimated from a societal perspective (to include costs borne by private hospitals and insurance providers, private patients and social care service providers). Resource use data will include a number of tests, treatments and investigations undertaken during in-hospital visits, the frequency and duration of in-hospital admissions, over the counter medication, lost productivity, social care services, as well as quantity and type of consumables and equipment used. Resource use data to capture patient-specific costs incurred will only be collected after discharge, using a resource use questionnaire that will be administered at 2, 4, 6, 12, 26 and 52 weeks. Resource unit costs will be derived from hospital finance departments and supplemented, where necessary, by information from published source including the Australian Bureau of Statistics and Australian Refined Diagnosis Related Groups (AR-DRG) cost weights. Confidence intervals will be presented around the incremental cost-effectiveness ratios and costeffectiveness acceptability curves for varying threshold values of cost-effectiveness will also be presented [37]. An assessment of the sensitivity of the results obtained to variation in measured resource use, effectiveness and/or unit costs will be undertaken using appropriate deterministic and probabilistic sensitivity analyses [38].

\section{Study duration}

The study accrual period will be approximately 12 months. Each individual will be followed for 12 months. 


\section{Sample size}

A sample size calculation based on achieving $90 \%$ power at two-tailed type 1 error rate of $5 \%$ identified that 110 participants (55 participants per group) would be required to detect $10^{\circ}$ of difference in ROM between the Maxm Skate program and standard care groups at three months after TKA assuming a within-group SD of $16^{\circ}$. The clinically significant difference in ROM of $10^{\circ}$ and SD was estimated from parameters described by Mockford et al. [16], in which the effect of a physiotherapy regimen on ROM was measured over a oneyear, post-TKA follow-up period. Furthermore, it is well established that daily activities, including climbing stairs and standing from a chair, require $90-120^{\circ}$ of flexion, kneeling and squatting $110-165^{\circ}$, and $135^{\circ}$ of flexion to lift from a bath [7-9]. However, patients rarely achieve $>120^{\circ}$ of flexion post TKA and $110^{\circ}$ of flexion is an optimal goal for rehabilitation following TKA [9-11]. Based on such awareness of requirements for daily activities, a $10^{\circ}$ difference in ROM may delineate between the ability to climb stairs or kneel down in TKA patients.

In order to account for a potential 5\% loss to follow-up, a total sample of 116 participants (58 per group) will be recruited.

\section{Statistical methods}

All analyses will be conducted on an intention-to-treat basis and results reported in accordance with CONSORT guidelines [39]. The primary endpoint is the ROM at three months and differences between groups will be assessed using an independent two-sample t-test with log-transformation of the outcome, if necessary, in order to meet the assumptions of normality. If normality is not achieved using transformation, then the Mann-Whitney $U$ test will be used. Differences between groups for the clinical performance tests (30-s Chair-stand and $4 \times 10 \mathrm{~m}$ Fast Paced Walk Test) and data collected on scales such as the OKS will also be assessed using a two-sample t-test or Wilcoxon Rank sum test if normality is not achieved. Baseline characteristics, including ROM, will be accounted for in a sensitivity analysis using mixed effects models that will also account for any missing data. Where $>10 \%$ of data are missing, multiple imputation using chained equations will also be used. Functional evaluations that are assessed at multiple time points will also be assessed using mixed effects models with an interaction term between group and visit used to determine differences in group effects across time. Two-sided hypothesis tests will be performed for each outcome with a type-1 error rate of alpha $=0.05$ used for determining statistical significance.

\section{Assessment of safety and criteria for study discontinuation}

The management of AEs that occur during this clinical trial will be in accordance with the guidelines of the approving Human Research Ethics Committee.

A safety analysis will be performed by a safety monitoring board that includes an independent orthopaedic field expert when $50 \%$ of the participants (58) reach the primary endpoint, at 12 weeks after TKA.

The primary criteria for discontinuation of the study will be poor outcomes, indicated by ROM. If > 18 participants $(30 \%)$ in the experimental group (MAXM Skate program) present a ROM of $<90^{\circ}$ at six weeks after TKA [40], the study will be discontinued. ROM assessments performed at six weeks will be conducted by both the study Physiotherapist at the six-week functional follow-up visit (specific to the study), as well as the treating orthopaedic surgeon at the routine six-week follow-up visit (routine clinical care at six weeks).

\section{Data management}

Clinical study data will be recorded directly on the Case Report Form (CRF) and will be completed for each participant enrolled into the clinical study. The Principal Investigator or nominated Co-Investigator will review, approve and sign/date each completed CRF series, attesting to the accuracy and authenticity of the data entered.

Clinical study records will be filed in the Clinical Study Master File in an organised way that will facilitate management of the clinical study, audit and inspection. The files will be retained securely before archive and then archived for a period of not less than 15 years to allow for audit and inspection by regulatory authorities upon request.

The Principal Investigator will permit direct access of the study monitors, the appropriate Human Research Ethics Committee and appropriate regulatory authorities to the study data when required.

\section{Ethics and dissemination}

This study received ethical approval from The Bellberry Human Research Ethics Committee on 24 September 2018. Any approved amendments made to the study protocol will be updated on the trial registry and the Therapeutic Goods Administration Clinical Trial Notification record, if appropriate.

Throughout the trial, investigators will endeavour to present preliminary findings at national and international orthopaedic conferences. At the conclusion of the trial, results will be prepared for presentation and publication in scientific journals. 


\section{Discussion}

With the increasing demand for TKA, further research must explore safe, optimal, cost-effective alternatives for physical rehabilitation following TKA. This singleblinded RCT will compare safety, efficacy and costeffectiveness of a novel home-based approach, the Maxm Skate rehabilitation program to standard rehabilitative care following TKA.

\section{Study limitations}

As is well discussed in the literature, there is potential for single-centre studies to have limited external validity. In the case of the present study, this may be considered a limitation given the enrolment of privately insured patients and the rehabilitation pathways offered to them in isolation, as compared to the rehabilitation pathways that may be available or typically selected in public healthcare centres. As such, in this study we are only capable of capturing the postoperative rehabilitation pathways that are routinely selected at Flinders Private Hospital. Interestingly, in Australia the mean discharge of privately insured TKA and THA patients to inpatient rehabilitation facilities is $40.1 \%$, compared to $20 \%$ in public patients [41]. As such, this study will capture the frequency, length and type of rehabilitation the study participants receive, in the hopes of improving transparency and generalisability of care. In addition, the study will also be recruiting a single surgeon's patients, which once again may reduce generalisability across surgeons within and across centres. Despite this, in order to reduce any potential bias associated with a single surgeon's patient cohort, the study orthopaedic surgeon is to be completely blinded to treatment allocation and, wherever possible, blinded to study participation altogether. Previous studies have addressed the heterogeneity of standard care options for rehabilitation between private hospitals in Australia [42] and this also presents in our study as a potential limitation. Further to this, there may also be a deal of heterogeneity across the Maxm Skate group participants, as despite their allocation and use of the Maxm Skate exercise program, should they feel it necessary to receive additional physiotherapy to assist in their recovery, they are able to do so. These variations in rehabilitative care are unavoidable to provide equal and consistent clinical care opportunities across all patients. In order to account for such heterogeneity in the rehabilitative pathway, a clear outline of the frequency, duration and type of treatment received by each participant will be documented at each follow-up visit with the Study Coordinator. Furthermore, at study conclusion, per-protocol analyses will be performed within the Maxm Skate group to distinguish any differences observed between participants receiving any physiotherapy care additional to the Maxm Skate exercise program. Such 'as-treated' grouping may assist in addressing the recognised limitation of participant receipt of additional care masking any potential benefit of the Maxm Skate exercise program. Despite the above recognised limitations of this study, we believe this RCT will provide sound evidence describing the potential differences observed over the course of different rehabilitative pathways of TKA patients in our centre.

\section{Trial status}

This publication is based on the study protocol version 08, dated 15 October 2018. Participant recruitment is anticipated to commence in October 2018, with an approximate recruitment period of 12 months. Details of the Maxm Skate study team are listed in Table 1.

Table 1 Maxm Skate Study team

\begin{tabular}{|c|c|c|}
\hline Name & Role on team & Affiliation \\
\hline Professor Jeganath Krishnan & Principle Investigator & $\begin{array}{l}\text { College of Medicine and Public Health, Flinders University, Adelaide, } \\
\text { South Australia, Australia; The International Musculoskeletal Research } \\
\text { Institute Inc., Adelaide, Australia }\end{array}$ \\
\hline Dr Matthew G. Liptak & Co-investigator; treating Orthopaedic Surgeon & Orthopaedic SA, Adelaide, Australia \\
\hline Annika Theodoulou & $\begin{array}{l}\text { Clinical Trial Coordinator (pre-recruitment [study } \\
\text { design and set-up]) }\end{array}$ & $\begin{array}{l}\text { College of Medicine and Public Health, Flinders University, Adelaide, } \\
\text { South Australia, Australia; The International Musculoskeletal Research } \\
\text { Institute Inc., Adelaide, Australia }\end{array}$ \\
\hline Dr Kristen Georgiou & Clinical Trial Coordinator (during recruitment) & $\begin{array}{l}\text { College of Medicine and Public Health, Flinders University, Adelaide, } \\
\text { South Australia, Australia; The International Musculoskeletal Research } \\
\text { Institute Inc., Adelaide, Australia }\end{array}$ \\
\hline Scott W. Hinrichs & Co-investigator; blinded physiotherapist & Flinders Private Hospital, Adelaide, Australia \\
\hline Dr Steve Saunders & $\begin{array}{l}\text { Physiotherapist; development of Maxm Skate } \\
\text { rehabilitation program }\end{array}$ & $\begin{array}{l}\text { Adjunct researcher - University of South Australia } \\
\text { Director - Saunders Sports and Spinal } \\
\text { Science and Medical Coordinator - Adelaide Football Club }\end{array}$ \\
\hline A/Prof Billingsley Kaambwa & Health Economist & $\begin{array}{l}\text { Health Economics Unit, College of Medicine and Public Health } \\
\text { Flinders University }\end{array}$ \\
\hline Prof Richard J. Woodman & Biostatistician & $\begin{array}{l}\text { Flinders Centre for Epidemiology and Biostatistics, College of } \\
\text { Medicine, Flinders University }\end{array}$ \\
\hline
\end{tabular}


This protocol publication has been prepared on the basis of the Standard Protocol Items: Recommendations for Interventional Trials (SPIRIT) guidelines. This SPIRIT Checklist is available in the Additional file 3.

\section{Additional files}

Additional file 1: Maxm Skate Rehabilitation Guide. (PDF 2897 kb)

Additional file 2: Outpatient Standard Care Physiotherapy Protocol (FPH). (DOCX $15 \mathrm{~kb}$ )

Additional file 3: SPIRIT Checklist. (DOC $123 \mathrm{~kb}$ )

\section{Abbreviations}

AE: Adverse event; App: iOS Application; AR-DRG: Australian Bureau of Statistics and Australian Refined Diagnosis Related Groups; CRF: Case Report Form; EQ - 5D 5L: Eurogol 5 Dimensional Health Survey - Level 5; FPH: Flinders Private Hospital; IOS: IPhone Operating System; KOOS: Knee Injury and Osteoarthritis Outcome Score; MVIC: Maximum voluntary isometric contraction; OARSI: Osteoarthritis Research Society International; OKS: Oxford Knee Score; PASS: Patient Acceptable Symptom State; QALY: Quality-adjusted life year; RCT: Randomised controlled trial; ROM: Range of motion; TKA: Total knee arthroplasty; UK: United Kingdom; VAS - Pain: Visual Analogue Scale for Pain

\section{Acknowledgments}

The authors gratefully acknowledge support provided through the South Australian Innovation Project Grant. We also thank Dr. Stephen J. Quinn for providing preliminary statistical assistance including an initial sample size calculation and Flinders Private Hospital for allowing the conduct of this study at their site.

\section{Funding}

The financial obligations of this study will be met through the following avenues:

- In-kind support for research related activities from the International Musculoskeletal Research Institute Incorporated Inc. (IMRI) which was supplemented by the South Australian Innovation Project Grant.

- In-kind support for physiotherapy and site resources from Flinders Private Hospital.

- In-kind support from Maxm Skate Pty Ltd. (provision of the Maxm Skate product).

- Maxm Skate Pty Ltd. provided financial support to meet the costs of the health economics evaluation, study insurance and provision of an IMRI researcher to assist with coordination of the study.

\section{Availability of data and materials}

Not applicable.

\section{Authors' contributions}

MGL was a major contributor to the study conception and will screen patients for eligibility once the study commences. AT developed the study protocol including conducting the literature review and prepared all study documentation including data collection forms and the ethics application documents. AT coordinated all study related-activities before study commencement and drafted the protocol manuscript. At the time of publication, AT was funded by the NIHR Oxford Biomedical Research Centre. The views expressed are those of the author and not necessarily those of the NHS, the NIHR or the Department of Health and Social Care. BK designed and will conduct the health economic evaluation of the trial. SS contributed to the design of the Maxm Skate rehabilitation program and functional evaluation of this trial. SWH assisted in the design and will conduct the functional evaluations for this trial. RJW will provide random sequence allocation codes for the trial, has reviewed the statistical analysis plan and will be in charge of all statistical aspects of the trial. JK contributed to the study design and will ensure the conduct of the trial and contribute to the data interpretation, presentation and publication following completion. All authors read and approved the final manuscript.

\section{Ethics approval and consent to participate}

This study received ethical approval from The Bellberry Human Research Ethics committee (HREC) on 24 September 2018. Study ID: 2016-03-221. Informed consent will be obtained from all study participants.

\section{Consent for publication}

Not applicable.

\section{Competing interests}

As the inventor of the Maxm Skate Device and package and Managing Director of Maxm Pty Ltd., MGL presents a conflict of interest to this research. The study has been designed to manage this conflict though blinding of treatment groups and limiting MGL's role to clinical responsibilities as the treating surgeon. MGL will conduct the screening for eligibility of all patients consented for elective primary total knee arthroplasty; however, he will not be involved in recruitment or collection of data, other than completing the operative data collection form. At completion of this trial, MGL will be involved in data presentation and publication; however, this will be in collaboration with the study investigators, clinical researcher, physiotherapists, health economist and biostatistician. Analysis and interpretation of functional data will be performed by physiotherapist SS with advice from statistician RW; analysis and interpretation of economics evaluations will be performed by economist BK with advice from RW; patient-reported outcome measures will be analysed and interpreted by the clinical researcher KG with advice from RW. Once these analyses are complete, they will be circulated to all investigators for input and overall interpretation as a complete study. It is only at this point that device developer MGL will contribute to interpretation, particularly regarding clinical meaningfulness of the findings. MGL will be involved in presentation and publication of study findings. SS declares that he holds equity in Maxm Pty Ltd. The remaining authors (AT, BK, SWH, RJW and JK) declare that they have no competing interests.

\section{Publisher's Note}

Springer Nature remains neutral with regard to jurisdictional claims in published maps and institutional affiliations.

\section{Author details}

${ }^{1}$ Orthopaedics SA, Adelaide, South Australia, Australia. ${ }^{2}$ The International Musculoskeletal Research Institute Inc., Adelaide, South Australia, Australia. ${ }^{3}$ College of Medicine and Public Health, Flinders University, Adelaide, Australia. ${ }^{4}$ NIHR Oxford Biomedical Research Centre, Oxford University Hospitals NHS Foundation Trust, Oxford, UK. ${ }^{5}$ Health Economics Unit, College of Medicine and Public Health, Flinders University, Adelaide, Australia. ${ }^{6}$ Saunders Sports and Spinal, Adelaide, Australia. ${ }^{7}$ Flinders Private Hospital, Adelaide, South Australia, Australia. ${ }^{8}$ Flinders Centre for Epidemiology and Biostatistics, College of Medicine, Flinders University, Adelaide, Australia.

Received: 3 October 2018 Accepted: 4 December 2018 Published online: 10 January 2019

\section{References}

1. Oatis CA, Li W, JM DR, Hoover MJ, Johnston KK, Butz MK, et al. Variations in delivery and exercise content of physical therapy rehabilitation following total knee replacement surgery: a cross-sectional observation study. Int J Phys Med Rehabil. 2014;Suppl 5:002.

2. Pozzi F, Snyder-Mackler L, Zeni J. Physical exercise after knee arthroplasty: a systematic review of controlled trials. Eur J Phys Rehabil Med. 2013;49(6): 877-92.

3. Bourne RB, Chesworth BM, Davis AM, Mahomed NN, Charron KD. Patient satisfaction after total knee arthroplasty: who is satisfied and who is not? Clin Orthop Relat Res. 2010;468(1):57-63.

4. Rutherford RW, Jennings JM, Levy DL, Parisi TJ, Martin JR, Dennis DA. Revision Total knee arthroplasty for arthrofibrosis. J Arthroplast. 2018; 33(75):S177-81.

5. Schroer WC, Berend KR, Lombardi AV, Barnes CL, Bolognesi MP, Berend ME, et al. Why are total knees failing today? Etiology of total knee revision in 2010 and 2011. J Arthroplast. 2013;28(8 Suppl):116-9.

6. Cheuy VA, JRH F, Paxton RJ, Bade MJ, Zeni JA, Stevens-Lapsley JE. Arthrofibrosis associated with total knee arthroplasty. J Arthroplast. 2017; 32(8):2604-11. 
7. Hefzy MS, Kelly BP, Cooke TD. Kinematics of the knee joint in deep flexion: a radiographic assessment. Med Eng Phys. 1998;20(4):302-7.

8. Mulholland SJ, Wyss UP. Activities of daily living in non-Western cultures: range of motion requirements for hip and knee joint implants. Int J Rehabil Res. 2001;24(3):191-8.

9. Rowe PJ, Myles CM, Walker C, Nutton R. Knee joint kinematics in gait and other functional activities measured using flexible electrogoniometry: how much knee motion is sufficient for normal daily life? Gait Posture. 2000; 12(2):143-55.

10. Bellemans J, Banks S, Victor J, Vandenneucker H, Moemans A. Fluoroscopic analysis of the kinematics of deep flexion in total knee arthroplasty. Influence of posterior condylar offset. J Bone Joint Surg (Br). 2002;84(1):50-3.

11. Fu H, Wang J, Zhang W, Cheng T, Zhang X. No clinical benefit of high-flex total knee arthroplasty. A meta-analysis of randomized controlled trials. J Arthroplast. 2015;30(4):573-9.

12. Naylor J, Harmer A, Fransen M, Crosbie J, Innes L. Status of physiotherapy rehabilitation after total knee replacement in Australia. Physiother Res Int. 2006;11(1):35-47.

13. Mistry JB, Elmallah RD, Bhave A, Chughtai M, Cherian JJ, McGinn T, et al. Rehabilitative guidelines after total knee arthroplasty: a review. J Knee Surg. 2016;29(3):201-17.

14. Peter WF, Nelissen RG, Vlieland TP. Guideline recommendations for postacute postoperative physiotherapy in total hip and knee arthroplasty: are they used in daily clinical practice? Musculoskeletal Care. 2014;12(3):125-31.

15. Australian Orthopaedic Association National Joint Replacement Registry (AOANJRR). Hip, Knee \& Shoulder Arthroplasty: 2017 Annual Report. Adelaide: $\mathrm{AOA} ; 2017$.

16. Mockford BJ, Thompson NW, Humphreys P, Beverland DE. Does a standard outpatient physiotherapy regime improve the range of knee motion after primary total knee arthroplasty? J Arthroplasty. 2008;23(8):1110-4.

17. Rajan RA, Pack Y, Jackson H, Gillies C, Asirvatham R. No need for outpatient physiotherapy following total knee arthroplasty: a randomized trial of 120 patients. Acta Orthop Scand. 2004;75(1):71-3.

18. Ko V, Naylor J, Harris I, Crosbie J, Yeo A, Mittal R. One-to-one therapy is not superior to group or home-based therapy after total knee arthroplasty: a randomized, superiority trial. J Bone Joint Surg Am. 2013;95(21):1942-9.

19. Florez-Garcia M, Garcia-Perez F, Curbelo R, Perez-Porta I, Nishishinya B, Rosario Lozano MP, et al. Efficacy and safety of home-based exercises versus individualized supervised outpatient physical therapy programs after total knee arthroplasty: a systematic review and meta-analysis. Knee Surg Sports Traumatol Arthrosc. 2017;25(11):3340-53.

20. Kramer JF, Speechley M, Bourne R, Rorabeck C, Vaz M. Comparison of clinicand home-based rehabilitation programs after total knee arthroplasty. Clin Orthop Relat Res. 2003:410:225-34.

21. Australian Orthopaedic Association National Joint Replacement Registry (AOANJRR). Hip, Knee and Shoulder Arthroplasty. Annual Report, 2018 Adelaide: AOA; 2018

22. Pua YH, Seah FJ, Seet FJ, Tan JW, Liaw JS, Chong HC. Sex differences and impact of body mass index on the time course of knee range of motion, knee strength, and gait speed after total knee arthroplasty. Arthritis Care Res. 2015;67(10):1397-405.

23. Gustavson AM, Wolfe P, Falvey JR, Eckhoff DG, Toth MJ, Stevens-Lapsley JE. Men and women demonstrate differences in early functional recovery after total knee arthroplasty. Arch Phys Med Rehabil. 2016;97(7):1154-62.

24. Dawson J, Fitzpatrick R, Murray D, Carr A. Questionnaire on the perceptions of patients about total knee replacement. J Bone Joint Surg Br. 1998; 80(1):63-9.

25. Roos EM, Roos HP, Lohmander LS, Ekdahl C, Beynnon BD. Knee Injury and Osteoarthritis Outcome Score (KOOS)--development of a self-administered outcome measure. J Orthop Sports Phys Ther. 1998;28(2):88-96.

26. Rabin R, de Charro F. EQ-5D: a measure of health status from the EuroQol Group. Ann Med. 2001;33(5):337-43.

27. Tubach F, Ravaud P, Baron G, Falissard B, Logeart I, Bellamy N, et al. Evaluation of clinically relevant states in patient reported outcomes in knee and hip osteoarthritis: the patient acceptable symptom state. Ann Rheum Dis. 2005;64(1):34-7.

28. Zhou Z, Yew KS, Arul E, Chin PL, Tay KJ, Lo NN, et al. Recovery in knee range of motion reaches a plateau by 12 months after total knee arthroplasty. Knee Surg Sports Traumatol Arthrosc. 2015;23(6):1729-33.
29. Holm B, Kristensen MT, Bencke J, Husted H, Kehlet H, Bandholm T. Loss of knee-extension strength is related to knee swelling after total knee arthroplasty. Arch Phys Med Rehabil. 2010;91(11):1770-6.

30. Lauermann SP, Lienhard K, Item-Glatthorn JF, Casartelli NC, Maffiuletti NA Assessment of quadriceps muscle weakness in patients after total knee arthroplasty and total hip arthroplasty: methodological issues. J Electromyogr Kinesiol. 2014;24(2):285-91.

31. Schache MB, JA MC, Webster KE. Lower limb strength following total knee arthroplasty: a systematic review. Knee. 2014;21(1):12-20.

32. Harikesavan K, Chakravarty RD, Maiya AG. Influence of early mobilization program on pain, self-reported and performance based functional measures following total knee replacement. J Clin Orthop Trauma. 2018. In press.

33. Hall M, Hinman RS, Wrigley TV, Kasza J, Lim BW, Bennell KL. Knee extensor strength gains mediate symptom improvement in knee osteoarthritis: secondary analysis of a randomised controlled trial. Osteoarthr Cartil. 2018; 26(4):495-500.

34. Skou ST, Wise BL, Lewis CE, Felson D, Nevitt M, Segal NA, et al. Muscle strength, physical performance and physical activity as predictors of future knee replacement: a prospective cohort study. Osteoarthr Cartil. 2016;24(8):1350-6.

35. Dobson F, Hinman RS, Roos EM, Abbott JH, Stratford P, Davis AM, et al. OARSI recommended performance-based tests to assess physical function in people diagnosed with hip or knee osteoarthritis. Osteoarthr Cartil. 2013; 21(8):1042-52.

36. Devlin N, et al. Valuing Health-Related Quality of Life: An EQ-5D-5L Value Set for England. London: Office of Health Economics; 2016.

37. Glick HA, Briggs AH, Polsky D. Quantifying stochastic uncertainty and presenting results of cost-effectiveness analyses. Expert Rev Pharmacoecon Outcomes Res. 2001;1(1):25-36.

38. Briggs $A H, O^{\prime} B r i e n ~ B J, ~ B l a c k h o u s e ~ G$. Thinking outside the box: recent advances in the analysis and presentation of uncertainty in cost-effectiveness studies. Annu Rev Public Health. 2002;23:377-401.

39. Moher D, Hopewell S, Schulz KF, Montori V, Gotzsche PC, Devereaux PJ, et al. CONSORT 2010 explanation and elaboration: updated guidelines for reporting parallel group randomised trials. BMJ. 2010;340:C869.

40. Schiavone Panni A, Cerciello S, Vasso M, Tartarone M. Stiffness in total knee arthroplasty. J Orthop Traumatol. 2009:10(3):111-8.

41. Royal Australasian College of Surgeons. Rehabilitation Pathways Following Hip and Knee Arthroplasty. North Adelaide: Royal Australasian College of Surgeons; 2018

42. Schilling C, Keating C, Barker A, Wilson SF, Petrie D. Predictors of inpatient rehabilitation after total knee replacement: an analysis of private hospital claims data. Med J Aust. 2018;209(5):222-7.

\section{Ready to submit your research? Choose BMC and benefit from:}

- fast, convenient online submission

- thorough peer review by experienced researchers in your field

- rapid publication on acceptance

- support for research data, including large and complex data types

- gold Open Access which fosters wider collaboration and increased citations

- maximum visibility for your research: over $100 \mathrm{M}$ website views per year

At BMC, research is always in progress.

Learn more biomedcentral.com/submissions 